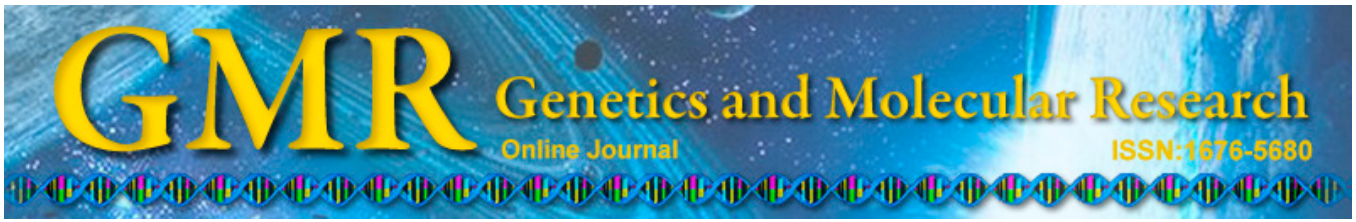

\title{
Inhibitory effects of spironolactone on myocardial fibrosis in spontaneously hypertensive rats
}

\author{
H. Zhao ${ }^{1,2}$, D.W. Gu' ${ }^{2}$, H.T. Li $^{2}$, Q.F. Ge ${ }^{2}$ and G.P. Li ${ }^{1}$ \\ ${ }^{1}$ Tianjin Key Laboratory of Ionic-Molecular Function of Cardiovascular Disease, \\ Department of Cardiology, Tianjin Institute of Cardiology, \\ Second Hospital of Tianjin Medical University, Tianjin, China \\ ${ }^{2}$ Department of Cardiology, \\ Affiliated Hospital of North China University of Science and Technology, \\ Tangshan, China \\ Corresponding author: G.P. Li \\ E-mail: tjcardio@126.com
}

Genet. Mol. Res. 14 (3): 10315-10321 (2015)

Received January 8, 2015

Accepted May 15, 2015

Published August 28, 2015

DOI http://dx.doi.org/10.4238/2015.August.28.17

\begin{abstract}
This study evaluated the inhibitory effects of spironolactone, a non-selective aldosterone receptor antagonist, on hypertensioninduced myocardial fibrosis. Collagen I and III contents was detected in the myocardial tissue of spontaneously hypertensive rats (SHRs) after spironolactone administration. Twenty male SHRs were assigned to the spironolactone group or control group $(\mathrm{N}=10$ each); 7 Wistar-Kyoto rats (WKY) were also used. Spironolactone dissolved in $\mathrm{ddH}_{2} \mathrm{O}$ was administered via gavage at a dosage of $20 \mathrm{mg} \cdot \mathrm{kg}^{-1} \cdot \mathrm{day}^{-1}$. Meanwhile, the control and WKY groups were administered equivalent volumes of $\mathrm{ddH}_{2} \mathrm{O}$ for 16 weeks. Western blotting was used to detect the contents of collagen I in myocardial tissue; observations were performed using polarizing microscopy, and the area integration and ratio of collagen I/ III were subsequently calculated. Compared to the WKY group, col-
\end{abstract}


lagen I synthesis was significantly higher in the control group (1.87 \pm 0.2 vs $1.21 \pm 0.7, \mathrm{P}<0.05)$. After 16 weeks of treatment, collagen I contents were significantly lower in the spironolactone group than in the control group $(1.42 \pm 0.05 v s 1.87 \pm 0.2, \mathrm{P}<0.05)$. The areas of collagen I and collagen I/III ratio were significantly smaller in the spironolactone group than in the control group $(6400 \pm 259$ vs $12,019 \pm 734$ pixels, $15.64 \pm 1.34$ vs $20.8 \pm 3.04$ pixels, respectively; $\mathrm{P}<0.05)$. However, there were no significant differences in the area of collagen III among the three groups. In conclusion, spironolactone improves myocardial collagen deposition, preventing myocardial fibrosis in SHRs.

Key words: Spontaneous hypertension rats; Myocardial fibrosis; Spironolactone; Collagen I; Collagen III

\section{INTRODUCTION}

The heart is the target organ directly involved in the progression of essential hypertension. Collagen deposition is generally considered the main causative factor of myocardial hypertrophy, myocardial fibrosis, myocardial tissue, and ion channel remodeling. The main treatment method is ACEI administration, which can improve and control the progression of myocardial fibrosis to protect the heart. The non-selective aldosterone receptor antagonist spironolactone can downregulate fibroblast collagen I mRNA expression (Bunda et al., 2007) and thus improve cardiac hypertrophy and collagen deposition in spontaneously hypertensive rats (SHRs) (Peng et al., 2002; Xu et al., 2008). Moreover, spironolactone can downregulate collagen mRNA expression (Matsumoto et al., 2004) in rats with myocardial infarction. Moreover, spironolactone improves the prognosis of heart failure and reduces serum markers of collagen I and III synthesis in patients with myocardial infarction or dilated cardiomyopathy (Zannad et al., 2000; Izawa et al., 2005). As serum marker levels and mRNA expression do not fully represent local collagen levels in myocardial tissue, SHRs were used in the present study. This study evaluated the effects of spironolactone on collagen I and III synthesis in the myocardial tissue of SHRs during myocardial fibrosis progression in order to provide a basis for clinical practice.

\section{MATERIAL AND METHODS}

\section{Experimental animals}

Twenty male 7-week-old SHRs weighing 120-150 g were provided by the Experimental Animal Institute of Chinese Academy of Medical Sciences. Seven male 7-week-old WistarKyoto rats (WKY group) weighing 120-150 g were purchased from Shanghai Slac Laboratory Animal Co., Ltd. SHRs were randomly assigned to the spironolactone group $(\mathrm{N}=10)$ or control group $(\mathrm{N}=10)$. Rats were fed a normal diet for 1 week before the experiment. At 8 weeks of age, the spironolactone group was administered spironolactone dissolved in $\mathrm{ddH}_{2} \mathrm{O}$ at 20 
$\mathrm{mg} \cdot \mathrm{kg}^{-1} \cdot \mathrm{day}^{-1}$ via gavage. Meanwhile, the group and WKY groups received equivalent volumes of $\mathrm{ddH}_{2} \mathrm{O}$ via gavage. Body weight was measured weekly, and the dosage was adjusted according to weight gain for 16 consecutive weeks. All rats were kept in a quiet rearing room with an artificially controlled 12-h light/dark cycle under constant temperature and humidity.

\section{Sample collection}

At 24 weeks of age, the rats were weighed and anesthetized by administering an intraperitoneal injection of $20 \%$ urethane at $1 \mathrm{~g} / \mathrm{kg}$. The chest was opened rapidly, and the heart was excised and washed thoroughly with precooled saline. Then, the heart was dried with filter paper, and the atrioventricular free wall and right ventricular free wall were cut along the atrioventricular ring to isolate the left ventricle. The tissue was subsequently cut into two pieces from the bottom of the left ventricular to the apex of the heart. After labeling, one piece was immediately frozen in liquid nitrogen for western blotting for collagen I, and the other was put into $10 \%$ neutral formalin fixative for histological examination.

\section{Main reagents}

Sodium dodecyl sulfate, trisbase, mercaptoethanol acrylamide, N'-methylene-bisacrylamide were obtained from the Blood Institute Technology Company of the Chinese Academy of Medical Sciences; Coomassie brilliant blue and R-250 Sirius Red were purchased from Sigma (San Francisco, CA, USA); bromphenol blue was purchased from Sangon Biotech (Shanghai, China); rabbit anti-fetal skin collagen I monoclonal antibodies were purchased from Calbiochem (Germany); pre-stained protein molecular weight standard markers were from Fermentas (Lithuania); and rat anti-GAPDH monoclonal antibody was purchased from Kangcheng Biological Engineering Co., Ltd. (Shanghai China).

\section{Myocardial tissue section and Picrosirius red staining}

Myocardial tissue was fixed in 10\% neutral-buffered formalin for $24 \mathrm{~h}$, dehydrated, treated with xylene, and embedded in paraffin. Sections $(5-\mu \mathrm{m}$ thick) were stained in lapis lazuli blue for $10 \mathrm{~min}$, washed three times with water for $5 \mathrm{~min}$, stained with picrosirius red for $30 \mathrm{~min}$, differentiated in absolute alcohol for $30 \mathrm{~s}$, cleared in xylene, and sealed with optical gum. After picrosirius red staining, nuclei were labeled green, collagen were red and other components were yellow under a light microscope, respectively; under a polarizing microscopy (The Nikon E600POL), type I collagen fibers were red and yellow, and type III collagen were green, and the Pixerra Penguin 150ESCCD2 ImagePro Plus 5.5 image analysis system was used to calculate the areas of type I and III collagen (in pixels) and the ratio of collagen I/III = total area of collagen I / total area of collagen III.

\section{Western blot analysis of collagen I and III in myocardial tissue}

Myocardial tissue samples (100 mg) were washed with phosphate-buffered saline, cut into $1-\mathrm{mm}^{3}$ pieces, and treated overnight with $1 \mathrm{~mL} 0.5 \mathrm{M}$ acetic acid for expansion. 
The samples were homogenized at $4^{\circ} \mathrm{C}$ and centrifuged at $10,000 \mathrm{rpm}$ for $25 \mathrm{~min}$. They were subsequently treated with pepsin and stirred for full digestion at $4^{\circ} \mathrm{C}$ for $6 \mathrm{~h}$. Tris was added ( $\mathrm{pH}$ 7.4) to terminate enzymolysis. The protein concentration was measured according to the Bradford method. SDS-PAGE and western blotting were performed according to the second edition of Molecular Cloning (Aambrook et al., 2005). The sample volume was $25 \mu \mathrm{L}$. GAPDH was selected as an internal reference, and ECL Western Blotting KIT was used for development. A computer automated image analysis program performed cumulative grayscale analysis on bands of collagen I, then checked them against the bands of the internal reference to obtain the results (i.e., grayscale of collagen I grayscale value of GAPDH).

\section{Statistical analysis}

Data were analyzed by SPSS version 10.0 and are reported as means \pm SD. Comparisons among groups were performed by ANOVA, and pairwise comparisons were made using the least significant difference $t$-test. The level of significance was set at $\mathrm{P}<0.05$.

\section{RESULTS}

During the experiment, one rat each in the spironolactone and control groups died.

\section{Western blotting}

After 16 weeks of treatment, collagen I expressions in cardiac muscle were significantly higher in the control group than in the WKY and spironolactone groups $(\mathrm{P}<0.05)$ (Table 1).

\section{Polarizing microscopy}

The areas of collagen I and III, and collagen I/III ratio were significantly higher in the control group than in the WKY and spironolactone groups (all $\mathrm{P}<0.05$ ). However, there was no significant difference among the three groups with respect to the area of or collagen III (Table 1 and Figure 1).

\begin{tabular}{|c|c|c|c|c|c|}
\hline \multirow{2}{*}{ Groups } & \multirow[t]{2}{*}{$\mathrm{N}$} & \multicolumn{2}{|c|}{ Western blot (\%) } & \multicolumn{2}{|c|}{ Polarizing light microscopy } \\
\hline & & Grayscale value of collagen I & Collagen I (pixels) & Collagen III (pixels) & Ratio of collagen I/III \\
\hline Spironolactone & 9 & $1.42 \pm 0.05^{\dagger}$ & $6,400 \pm 259^{\dagger}$ & $368.3 \pm 30.20$ & $15.64 \pm 1.34^{\dagger}$ \\
\hline Control & 9 & $1.87 \pm 0.20 *$ & $12,019 \pm 734^{*}$ & $481.6 \pm 32.40$ & $20.80 \pm 3.04 *$ \\
\hline WKY & 7 & $1.21 \pm 0.70$ & $4,681 \pm 435$ & $406.2 \pm 45.30$ & $13.33 \pm 2.00$ \\
\hline F value & & 4.869 & 7.581 & 2.29 & 5.617 \\
\hline P value & & 0.013 & 0.014 & 0.126 & 0.003 \\
\hline
\end{tabular}

${ }^{\dagger} \mathrm{P}<0.05$ vs control group; $* \mathrm{P}<0.05$ vs WKY group. WKY $=$ Wistar-Kyoto rats. 

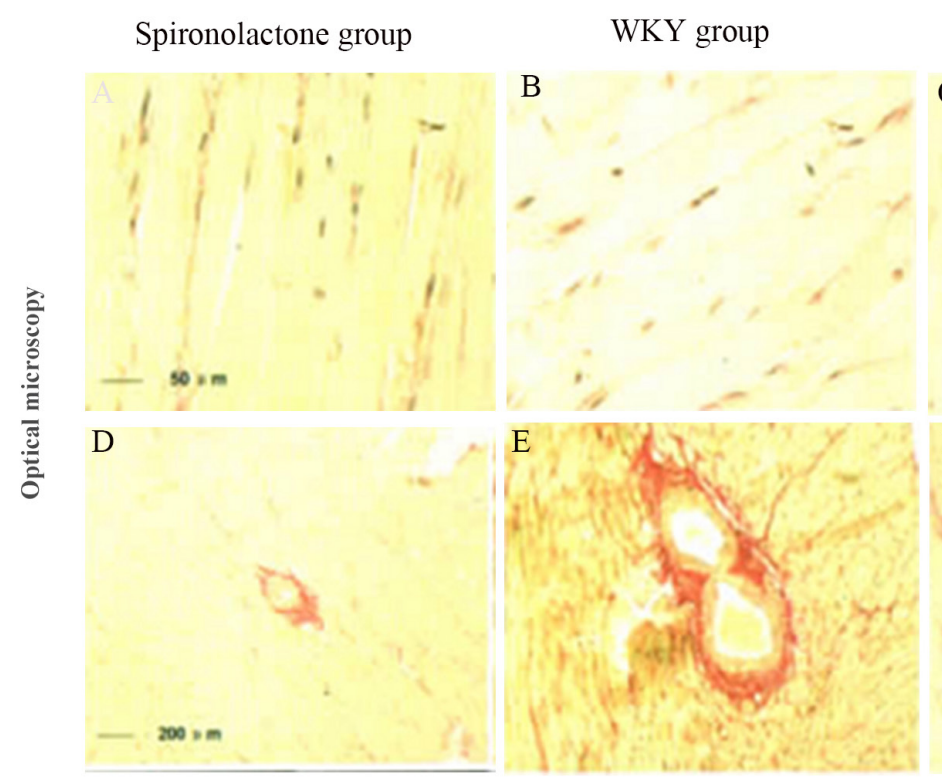

Control group
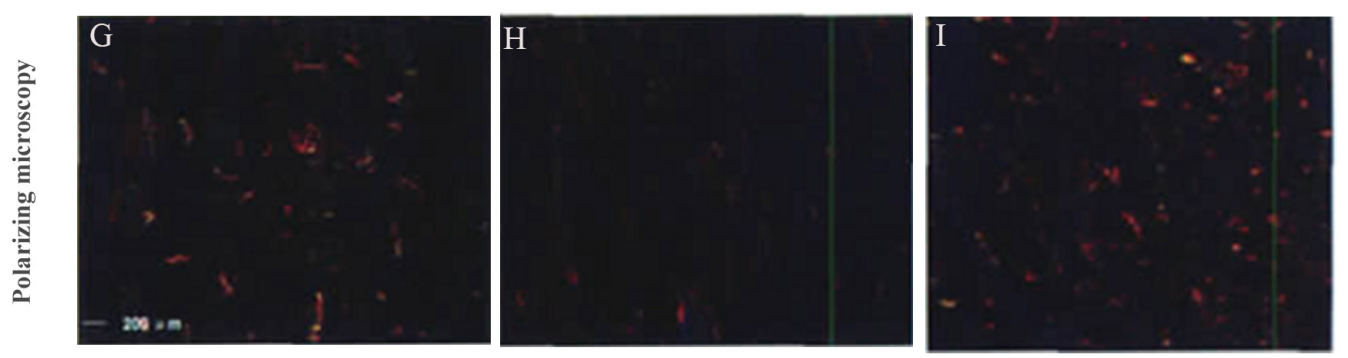

Figure 1. Numbers of cardiomyocytes in the left ventricle were examined using picrosirius red staining. Nuclei, collagen, and other components are green, red, and yellow, respectively. On polarization microscopy, type I collagen appears red and yellow, while type III collagen appears green. WKY = Wistar Kyoto rats.

\section{DISCUSSION}

SHRs are an experimental model of chronic hypertension. Yu et al. (1999) report that the blood pressure of SHRs often starts to increase at 6 weeks of age; however, unstable perivascular fibrosis emerges without affecting myocardial interstitial tissue. Blood pressure continues to increase in the 14th week (i.e., "increased period") when the level of myocardial hydroxyproline increases. When entering the 24th week, blood pressure stabilizes and myocardial interstitial fibrosis develops gradually. The present study aimed to determine the effects of spironolactone on myocardial collagen during the progression of essential hypertension. Therefore, we selected 7-week-old SHRS as our experimental subjects, and fed with normal diet for 1 week. The tratment was sustained for 16 weeks and stopped at the 24th week. The main side effects of spironolactone, when used as a nonselective aldosterone receptor antagonist, are breast development and dose-dependent sexual disturbance. Spironolactone can be administered at a dose of $12.5-25.0 \mathrm{mg} /$ day to effectively 
block aldosterone receptor without affecting hemodynamics but can cause hyperkalemia at a daily dose exceeding $50.0 \mathrm{mg}$ (Howard et al., 2006). Therefore, by referring to the clinical dose, we administered spironolactone at $20 \mathrm{mg} \cdot \mathrm{kg}^{-1} \cdot \mathrm{day}^{-1}$. Unlike angiotensin-converting enzyme inhibitors, spironolactone can prevent myocardial fibrosis in 22-month-old normal rats and can thus resist degenerative heart changes caused by aging (Lacolley et al., 2001). Therefore, the anti-myocardial fibrosis mechanism of spironolactone is attracting increasing attention. Interstitial myocardial tissue mainly consists of type I and III collagen. The main histopathological characteristics of hypertension are myocardial hypertrophy, deposition of type I collagen, the type and distribution of collagen varies, and the ratio of type I/III increases (Pauschinger et al., 1999). Because of the relative thickness of type I collagen, which plays a role in resisting tension and maintaining ventricular morphology, pressure exceeding $100 \mathrm{mmHg}$ can expand the ventricular wall by inhibiting the effects of collagen in a resting heart (Factors et al., 1988). Owing to its slimness, collagen III contributes to the features of myocardial extension and resilience. Left ventricular diastole including the early rapid filling phase is a process of active myocardial relaxation, while the late passive filling phase depends on the elasticity of the myocardium.

The results of the present study show that spironolactone decreased the quantities of collagen I as well as the ratio of collagen I/III, but had no significant effects in the myocardial tissue of SHRs. Collagen I and III were stained by picrosirius red and observed under a polarizing microscope to calculate their ratio. Under light microscopy, the collagen fibers were labeled red, nuclei were green and other components were yellow. Through polarizing microscope, collagen type I presented a yellow, orange, or red color, while collagen type III appeared green. Thus, collagen I and III can be observed simultaneously on one slide (Junqueiral et al., 1978).

Furthermore, the mRNA transcription of collagen occurs in fibroblasts, and the translated peptides become pre-collagen through hydroxylation and glycosylation. Precursor sequences of procollagen are cut off by a relative enzyme before a crosslinking reaction occurs to form procollagen, which gathers to form collagen fibers. Research on the effects of spironolactone on myocardial structure in essential hypertension, especially on collagen type and distribution, as well as its regulatory mechanism is of great importance.

\section{Conflicts of interest}

The authors declare no conflict of interest.

\section{ACKNOWLEDGMENTS}

Research subsidized as part of the "Study on the relationship between TNF $\alpha$ expression for antifibrotic effects of spironolactone in preventing hypertensive myocardial fibrosis and I, III collagen type" (\#12140209A-18).

\section{REFERENCES}

Aambrook J, Maniatis T and Fritsch EF (2005). Molecular Cloning: A Laboratory Manual. 2nd edn. Cold Spring Harbor Laboratory Press, Cold Spring Harbor, New York.

Bunda S, Liu P, Wang Y, Liu K, et al. (2007). Aldosterone induces elastin production in cardiac fibroblasts through activation of insulin-like growth factor-I receptors in a mineralocorticoid receptor independent manner. Am. $J$. Pathol. 171: 809-819. 
Delcayrec and Silvestrej S (1999). Aldosterone and the heart: towards a physiological function? Cardiovasc. Res. 43: 7-12. Factors M, Flomenbaum M, Zhaom J, Eng C, et al. (1988). The effects of acutely increased ventricular cavity pressure on intrinsic myocardial connective tissue. J. Am. Coll. Cardiol. 12: 1582-1589.

Howard PA, Cheng JW, Crouch MA, Colucci VJ, et al. (2006). Drug therapy recommendations from the 2005 ACC/AHA guidelines for treatment of chronic heart failure. Ann. Pharmacother. 40: 1607-1617.

Izawa H, Murohara T, Nagata K, Isobe S, et al. (2005). Mineralocorticoid receptor antagonist ameliorates left ventricular diastolic dysfunction and myocardial fibrosis in mildly symptomatic patients with idiopathic dilated cardiomyopathy: A pilot study. Circulation 112: 2940-2945.

Junqueiral C, Cossermelli W and Brentani R (1978). Differential staining of collagens type I, II and III by Sirius red and polarization microscopy. Arch. Histol. Jpn. 41: 267-274.

Lacolley P, Safarm E, Lucet B, Ledudal K, et al. (2001). Prevention of aortic and cardiac fibrosis by spironolactone in old normotensive rats. J. Am. Coll. Cardiol. 37: 662-667.

Matsumoto R, Yoshiyama M, Omura T, Kim S, et al. (2004). Effects of aldosterone receptor antagonist and angiotensin type 1 receptor blocker on cardiac transcriptional factors and mRNA expression in rats with myocardial infarction. Circ. J. 68: 376-382.

Pauschinger M, Knopf D, Petschauer S, Doerner A, et al. (1999). Dilated cardiomyopathy is associated with significant changes in collagen type I/III ratio. Circulation 99: 2750-2756.

Peng YP, Jiang SS and Chen RH (2002). Effects of angiotensin 1 type receptor antagonist and aldosterone receptor antagonist reverse myocardial remodeling on hypertensive rats. Zhongguo Dongmai Yinghua Zazhi 10: 408-410.

Xu M, Yang HD, Min XW and Li DF (2008). Spironolactone improves myocardial fibrosis in spontaneously hypertensive rats. Zhongguo Bingli Shengli Zazhi 24: 1281-1285.

Yu XJ, Qi WH and Gu DH (1999). Dynamic changes of left ventricular hypertrophy and myocardial fibrosis in the spontaneously hypertensive rats. Zhonghua Gaoxueya Zazhi 7: 159-162.

Zannad F, Alla F, Dousset B, Perez A, et al. (2000). Limitation of excessive extracellular matrix turnover may contribute to survival benefit of spironolactone therapy in patients with congestive heart failure: Insights from the randomized aldactone evaluation study (RALES). Circulation 102: 2700-2706. 